\title{
Voix africaines au Forum social mondial de Nairobi. Les chemins transnationaux des militantismes africains
}

Marie-Emmanuelle Pommerolle et Johanna Siméant

\section{(2) OpenEdition Journals}

Édition électronique

URL : http://journals.openedition.org/conflits/13423

DOI : $10.4000 /$ conflits. 13423

ISSN : 1777-5345

Éditeur :

CCLS - Centre d'études sur les conflits lilberté et sécurité, L'Harmattan

Édition imprimée

Date de publication : 20 juillet 2008

Pagination : 129-149

ISBN : 978-2-296-05909-2

ISSN : 1157-996X

Référence électronique

Marie-Emmanuelle Pommerolle et Johanna Siméant, « Voix africaines au Forum social mondial de Nairobi. Les chemins transnationaux des militantismes africains », Cultures \& Conflits [En ligne], 70 | été 2008, mis en ligne le 04 janvier 2010, consulté le 30 mars 2021. URL : http:// journals.openedition.org/conflits/13423; DOI : https://doi.org/10.4000/conflits.13423 


\title{
Voix africaines au Forum social mondial de Nairobi. Les chemins transnationaux des militantismes africains
}

\author{
Marie-Emmanuelle POMMEROLLE
}

Marie-Emmanuelle Pommerolle est mâtre de conférences en sciences politiques à l'université des Antilles et de la Guyane. Elle est chercheuse au Centre de recherche sur les pouvoirs locaux de la Caraïbe (CRPLC). Ses travaux portent sur la sociologie des mobilisations en Afrique (Kenya et Cameroun) et plus précisément sur les effets de l'extraversionsur le militantisme africain.Email : mepommerolle@free.fr

\section{Johanna SIMÉANT}

Johanna Siméant est professeur de sciences politiques à l'université Paris-I (CRPS) et membre de l'Institut universitaire de France. Elle travaille sur les mobilisations, l'action humanitaire et l'internationalisation du militantisme. Elle a notamment publié La Cause des sans-papiers, Paris, Presses de Sciences-Po, 1998 et, avec Pascal Dawvin et CAHIER, Le Travail humanitaire, Paris, Presses de Sciences-Po, 2002 et a dirigé avec Marc Le Pape et Claudine Vidal, Crises extrêmes. Face aux massacres, guerres et génocides, Paris, La Découverte, 2006.Email: jsimeant@univ-paris1.fr

19 janvier 2007, dans le confortable hôtel Six-eighty, au centre de Nairobi. C'est la soirée du CRID, le Centre de recherche et d'informations pour le développement, un collectif de cinquante-quatre associations de solidarité internationale qui constitue la principale structure de coordination des organisations françaises au Forum social mondial (FSM). Alors que la soirée se poursuit et que chacun présente son organisation et «ses partenaires ", tout à coup, un groupe de jeunes Kenyans, aidés par le groupe des No Vox, brandit des pancartes. Une jeune femme kenyane prend la parole au nom du People's Parliament, une organisation dont elle explique qu'elle n'a pas été suffisamment associée au FSM et au travail du comité local d'organisation. Wangui Mbatia évoque le prix de la participation au 
FSM (500 shillings kenyans, soit, explique-t-elle, une semaine de nourriture pour une famille pauvre). La jeune juriste, comme on l'apprendra par la suite, incarnera tout au long du forum la contestation d'une partie des militants kenyans à l'égard d'un forum souvent dénoncé comme "une conférence internationale de plus». Pendant tout le forum également se posera la question de la représentativité du People's Parliament : des rumeurs et insinuations circulent sur sa responsable, qui a étudié aux Etats-Unis, et que l'on retrouvera quelques mois plus tard aux protestations contre le G8 de Rostock. Mais la question est plus générale : qui a le droit de parler au nom des pauvres du Kenya et de l'Afrique lors des grands événements militants internationaux ?

20 janvier 2007, Nairobi, Uhuru Park, sur l'estrade, face à une colline qui donne aux lieux un aspect d'amphithéâtre. Cérémonie d'ouverture du FSM. Le public est très bigarré et compte beaucoup plus de Kenyans (l'entrée est libre) que le reste du FSM. Après les interventions très acclamées de Wahu Kaara, Valérie Traoré et Chico Whitaker, on annonce le secrétaire du Forum social africain. Taoufik Ben Abdallah arrive sur scène : «Hello, Viva Africa Viva! ». Très peu d'applaudissements l'accueillent et l'ensemble de son discours est reçu de façon extrêmement tiède, comme si le public était déconcerté que ce Tunisien ${ }^{1}$ à la peau claire parle au nom de l'Afrique.

Du 21 au 24 janvier 2007, en banlieue de Nairobi, dans des tribunes du stade de Kasarani aménagées pour recevoir les ateliers du FSM. Dans ceux consacrés à l'annulation de la dette, organisés par Jubilee South et d'autres organisations, le public africain est très minoritaire. Pourtant, sur cette thématique qui concerne l'Afrique au premier chef, la tribune voit défiler de nombreux militants africains : Viktor Nzuzi, de la République démocratique du Congo, lié aux réseaux du CADTM (Comité pour l'annulation de la dette du Tiers-Monde), l'économiste sénégalais Demba Moussa Dembelé, l'Ivoirien Diakalia Ouattara, ou encore la militante kenyane Wahu Kaara. Ces trois derniers sont membres du Forum social africain. Plus loin, dans l'atelier consacré au pastoralisme, seules quatre des soixante-dix personnes de la salle sont blanches, au milieu d'une audience où l'on remarque des personnes en tenue massaï ou encore des Ethiopiens.

Ces scènes militantes, toutes observées directement, se sont tenues au même endroit, mais ne racontent pas le même Forum social mondial. Elles montrent que, même dans le seul cadre d'observation d'un Forum social mondial, l'internationa- 
lisation du militantisme renvoie à des réalités multiples en fonction des canaux par lesquels les militants voyagent et protestent dans des lieux internationalisés.

Pourquoi observer en particulier ce Forum social mondial ? Tout d'abord, parce que si les autres FSM se sont tenus au Sud, il s'agit du premier FSM qui s'est tenu en Afrique, si l'on excepte le Forum social polycentrique de janvier 2006 dont une des émanations s'était tenue à Bamako. Or, bien qu'il existe une littérature désormais importante sur ces rassemblements protestataires transnationaux, et notamment des enquêtes sociologiques approfondies sur la participation à ces forums ${ }^{2}$, peu de recherches se sont intéressées à la localisation - géographique et symbolique - de ces forums. A Nairobi, les organisateurs du FSM n'ignoraient pas l'enjeu qu'il y avait à incarner l'Afrique dans ce forum, à faire entendre les voix africaines, d'autant que l'Afrique est perçue comme le continent le plus victime de la mondialisation. Réfléchir à l'Afrique au FSM de Nairobi, c'est à la fois réfléchir à l'émergence d'un altermondialisme africain, incarné entre autres par le Forum social africain (FSA), mais aussi à la multiplicité des réseaux africains transnationalisés et aux tensions entre ces derniers, ainsi qu'à la relation complexe qu'ils entretiennent à l'égard des militants du Nord ou d'autres Suds (Asie et Amérique latine). C'est, enfin, réfléchir à l'utilisation et au renouveau des thématiques afrocentriques, anti-impérialistes et anticoloniales.

Observer le FSM à Nairobi du point de vue du Sud - en particulier de l'Afrique - et de sa participation, c'est aussi un moyen d'éviter certains travers d'une sociologie des mouvements sociaux transnationaux, encore aujourd'hui majoritairement centrée sur la société civile du $\operatorname{Nord}^{3}$ ou qui, dans le meilleur des cas, traite de campagnes transnationales concernant des thèmes qui touchent le Sud (barrages, travail des enfants, etc.), mais majoritairement animées par des militants du Nord. Si l'existence d'un militantisme transnational du Sud est reconnue, elle est le plus souvent envisagée comme une adaptation ou une appropriation de dynamiques extérieures ${ }^{4}$. Les explications binaires de ce militantisme (voyant dans ce dernier soit une société civile émergente sui generis, soit, à l'inverse, les «valets » ou les « compradores 5 » d'un Nord toujours porté

2. Agrikoliansky E., Sommier I. (dirs.), Radiographie du mouvement altermondialiste, Paris, La Dispute, 2005 ; Della Porta D., Tarrow S. (eds.), Transnational Protest and Global Activism, Lanham, MD, Rowman \& Littlefield, coll. "People, Passions and Power", 2005.

3. Malgré quelques exceptions, voir notamment Wood L.J., "Bridging the chasms: the case of peoples' global action", in Bandy J., Smith J., Coalitions Across Borders. Transnational Protest and the Neoliberal Order, Lanham, MD, Rowman \& Littlefield, 2005, pp. 95-117 ; Rothman F.D., Oliver P., "From local to global: the anti-dam movement in Southern Brazil, 1979-1992", in Smith J., Johnston H. (eds.), Globalization and Resistance Transnational Dimensions of Social Movements, New York, Rowman \& Littlefield, 2002, pp. 115-132.

4. Wing S.D., "Women activists in Mali. The global discourse on Human Rights", in Desai M., Naples N.A. (eds), Women's Activism and Globalization. Linking Local Struggles and Transnational Politics, New York, Routledge, 2002, pp. 172-185.

5 . Ce terme, désignant à l'origine des intermédiaires commerciaux, fut particulièrement employé dans la sociologie de la dépendance pour stigmatiser les bourgeoisies compradores des pays victimes de l'impérialisme, et ayant structurellement intérêt à ce dernier du fait de leur position d'intermédiaires. 
au paternalisme), ne suffisent pas. Dans un espace altermondialiste qui cherche justement à les euphémiser, il importe de penser les hiérarchies, les conflits, voire tout simplement la division du travail au sein du militantisme transnational. Cela suppose de prêter attention aux conditions sociales et matérielles du militantisme ${ }^{6}$. On étudiera comment des militants africains ont réussi à participer au FSM de Nairobi et quels sont les conflits autour de la prise de parole sur l'Afrique. Plusieurs éléments s'inscrivent ici dans une histoire longue de l'extraversion militante ${ }^{7}$ en Afrique : débats sur le patronage militant du Nord, comme lors des luttes anti-coloniales, ou encore sur l'usage des « traditions » dans les luttes émancipatrices.

Cet article s'appuie sur une enquête collective réalisée à Nairobi en janvier $2007^{8}$ : une équipe de vingt-trois Français et douze Kenyans a mené des observations dans plus de cent cinquante ateliers du FSM. S’y sont ajoutés près de cent cinquante entretiens auprès de militants africains au forum ${ }^{9}$. L'enquête avait pour but d'observer la composition du public, le contenu et la forme des débats, la façon dont on parlait de l'Afrique. Les entretiens portaient sur la biographie des enquêtés et les aspects pratiques de leur participation au FSM (financement de leur voyage, etc.). Cette enquête a été complétée par la lecture d'articles de presse, de commentaires et de compte rendus des acteurs du FSM (le plus souvent via des listes de diffusion électronique). La pratique au sein de l'équipe de nombreux debriefings (pendant l'enquête) et relectures croisées explique le choix de ne pas recourir au «quantitatif masqué » à partir de ce matériau : plusieurs affirmations généralisantes dans ce texte s'appuient sur le fait qu'aucun membre de l'équipe ne les a contredites sur la base de ses propres observations.

Cette réflexion, qui s'articule autour des conditions matérielles et symboliques du militantisme, se déploie en trois temps. Une première partie s'intéresse à la participation des Africains au forum, en insistant sur les controverses autour de la représentativité des participants et les modes concrets de participation à ce

6. Comme cela a été fait par exemple à propos des conditions concrètes d'internationalisation des syndicalistes européens : Wagner A.-C., «Des syndicalistes européens. Les conditions sociales et institutionnelles de l'internationalisation des militants syndicaux ", Actes de la recherche en sciences sociales, 2004, $\mathrm{n}^{\circ} 155$, pp. 12-33.

7. Bayart, J.-F., «L'Afrique dans le monde : une histoire d'extraversion », Critique internationale, ${ }^{\circ} 5,1999$, pp. 97-120.

8. Dans le cadre d'un contrat de l'Agence nationale de la recherche intitulé «Causes africaines. Mobilisations, courtage international et luttes sur les formes légitimes du gouvernement de l'Afrique », consacré aux extraversions militantes.

9. En plus des deux auteurs, l'équipe était composée de : Thomas Atenga, Julie Aubriot, Xavier Audrain, Evelyn Awino, Marie Baget, Florence Brisset, Dominique Cardon, Dominique Connan, Camille Le Coq, Pascal Dauvin, Mathilde Debain, Sara Dezalay, Oita Etyang, Nicolas Haeringer, Idris Irshad, Fernando Isern, Lizz Kariuki, Lilian Kayaro, Fanny Laredo, Alphonse Maindo, Lilian Mathieu, Charles Mutua, Mwadzoya Mwandeje, Marame Ndour, John Ndung'u, Ayito Nguema, Margaret Njeru, Nicholas Odoyo, Vincent Opondo, Andrew Otieno Aura, Benjamin Osiemo, Samadia Sadouni, Nedjib Sidi Moussa, Guillaume Thiery, Nathanaël Tstotsa, Leonard Wambaya. Qu'ils soient ici remerciés de leur contribution. 
rassemblement. Nous nous arrêtons ensuite sur la formation d'un espace militant altermondialiste en Afrique en montrant les clivages, sociaux et symboliques, qui le structurent et l'animent. Enfin, nous observons combien la capacité et la légitimité à parler « au nom de l'Afrique » sont discutées et mises en scène.

\section{La participation africaine à un FSM en Afrique}

A l'automne 2006, on ne compte, au sein du comité international du FSM, que $8 \%$ d'organisations possédant un siège en Afrique ${ }^{10}$. L'intégration de délégués africains au FSM (entendus ici comme des personnes de nationalité africaine résidant en Afrique), a donc été vécue comme une nécessité afin que le FSM puisse continuer à se dire « mondial », d'où aussi le choix de l'Afrique pour accueillir cette septième édition du forum.

\section{Chiffres et controverses autour de la participation africaine an FSM}

Si la mesure de la participation au FSM doit être prise avec prudence, comme dans toute quantification de l'activité politique par ses acteurs, c'est bien parce que ces chiffres représentaient un enjeu, en termes de mesure et de représentativité du forum. Cependant, même les chiffres officiels les plus cités, probablement surévalués (45000 inscrits et 50000 participants pendant un ou deux jours), contrastent avec les 100000 attendus initialement ou présents à Porto Alegre en 2005, lors du dernier forum ${ }^{11}$. Parmi ces 45000 inscrits, $60 \%$ des participants sont africains selon les registres d'inscription (mais la réalité est sûrement moindre) ${ }^{12}, \mathrm{du}$ "jamais $v u$ » néanmoins selon les « anciens » du FSM. On aurait compté entre 400 et 1000 Tanzaniens, entre 50 et 120 Congolais (RDC), entre 25 et 50 Maliens, plus de 200 Sénégalais, 150 Soudanais, 360 Africains du Sud, le même nombre d'Ougandais ${ }^{13}$. On note ainsi une forte participation de ressortissants de pays dans lesquels les réseaux altermondialistes sont les plus anciens (Afrique de l'Ouest), mais aussi de Sud-africains (dont la tradition militante sera largement démontrée pendant le FSM), et de ressortissants d'Afrique de l'Est, venus en nombre important du fait de la proximité géographique. Ces chiffres, même imprécis, laissent entrevoir une participation importante d'Africains qui ne constituent d'habitude qu'une minorité dans les Forums sociaux mondiaux. Aux alentours de 30 en 2001 à Porto Alegre, ils auraient été 200 en 2002 et bien

10. Voir : http://www.forumsocialmundial.org.br/main.php?id_menu=3_2_18cd_language=3 (consulté le 22 novembre 2007).

11. Le Draft final report du Comité d'organisation donne, pour sa part, le chiffre de 74309 inscrits. Il est disponible sur :

http://www.africansocialforum.org/english/fsm/Kenya2007/report.htm (consulté le 22 novembre 2007)

12. Comité d'organisation, ibid. Il s'agit ici de $60 \%$ du total, sachant que $15 \%$ n'ont pas déclaré leur région d'origine.

13. Les chiffres concernant les délégués de nationalités autres qu'africaines sont les suivants: entre 1000 et 1500 Indiens, 150 Pakistanais, 600 Etats-uniens, 400 Brésiliens, 20 Haïtiens, 1000 Français, 400 Italiens, 240 Belges, 150 Espagnols, 50 Britanniques. 
plus en 2003. Ces chiffres sont comparables à l'affluence au Forum social africain (FSA), première émanation régionale du FSM : 200 délégués africains se sont retrouvés à Bamako en 2002, le même nombre à Addis Abeba en 2003 et entre 300 et 650, selon les estimations, à Lusaka en 2004.

La participation des Kenyans a été l'objet d'une controverse avant même l'ouverture du FSM ${ }^{14}$, et plus encore pendant et après le forum ${ }^{15}$. Pour nombre de militants altermondialistes, elle aurait été «moins bonne » que celle des nationaux, en Inde ou au Brésil ${ }^{16}$, même si elle semble avoir constitué $90 \%$ de la participation africaine ${ }^{17}$. La raison principale en serait le prix d'entrée fixé d'abord à 500 shillings kenyans (7 dollars US), puis divisé par dix après de vives protestations. Des groupes de militants (kenyans, sud-africains, français et japonais) ont cependant tenté de forcer les grilles dès le deuxième jour du FSM pour réclamer l'entrée libre et gratuite pour tous ; ils ont ensuite fait le siège des bureaux des organisateurs kenyans. Si ces obstacles matériels sont indéniables, plusieurs habitués des FSM, ainsi que les organisateurs kenyans incriminés, ont noté après coup que la participation des plus pauvres n'est jamais facile 18 - certains membres d'ONG ironisant en plus sur la tendance à se faire une représentation enchantée de la participation des plus pauvres. C'est plus globalement le manque de communication autour de l'événement et l'isolement du site qui ont donné cette impression d'une mobilisation peu ancrée dans la vie quotidienne des habitants de Nairobi, dont un tiers, vivant dans des bidonvilles, est pourtant particulièrement affecté par les problèmes soulevés par le forum. C'est avant tout l'Eglise catholique et les réseaux œcuméniques qui ont réussi à mobiliser des habitants des quartiers les plus pauvres en inscrivant 4000 d'entre eux au FSM. L'Eglise est en effet l'un des acteurs sociaux les plus présents dans les zones populaires et a créé de longue date des liens avec les ONG kenyanes les mieux connectées au militantisme international ${ }^{19}$.

\section{Participation, extraversion et internationalisation des délégués africains}

Les plus démunis ne sont pas les seuls à avoir été subventionnés pour participer au FSM. L'ensemble des délégués africains interrogés sur le financement de

14. La plate-forme œcuménique (Caritas et All African Church Conferences) avait envoyé une lettre au comité d'organisation en novembre 2006 demandant de baisser le tarif d'entrée à 40 shillings kenyans $(0,60$ dollars US) et de créer un fonds de solidarité afin de couvrir les frais de ceux qui ne pourraient pas s'en acquitter.

15. Pour plus de détails sur les significations de cette controverse, voir Haeringer N., Pommerolle M.-E., «Le FSM à l'épreuve de l'extraversion : les controverses au forum de Nairobi », communication présentée à la journée d'étude : "Un FSM en Afrique - Nairobi 2007. Causes africaines et extraversions militantes », Paris, 5 octobre 2007.

16. Gus Massiah cité dans le «Compte rendu de la journée d'évaluation du 17 février 2007 », organisée par la délégation du CRID au FSM 2007.

17. Comité d'organisation, op. cit.

18. Massiah G., op. cit.

19. Voir par exemple : Orvis S., "Kenyan civil society : bridging the urban-rural divide?”,Journal of Modern African Studies, vol.41, n², 2003, pp. 247-268. 
leurs billets d'avion dans le cadre de notre enquête a reçu l'aide d'une organisation du Nord. Le CRID a permis, notamment grâce à un financement du ministère français des Affaires étrangères, de payer le voyage et le séjour de 47 " partenaires » des organisations françaises de la coordination, dont 19 délégués africains. C'est le cas également de l'EED (Evangelische Entwicklungsdienst), des diverses délégations de la Caritas, d'Action Aid, d'Oxfam, et de la Croix-Rouge, de Brot für die Welt, de la Friedrich Ebert Stiftung, de la CGT, ainsi que d'organisations financièrement plus modestes, comme Via Campesina ou le CADTM. Ce patronage extérieur indispensable à la venue des militants africains existe depuis les débuts de la participation africaine au FSM 20.

Les objectifs de ce financement sont multiples pour les organismes du Nord. Contre l'image que le FSM tente de donner de lui-même (celle d'un lieu d'échange et de discussion entre participants non préalablement connectés), certains ateliers sont davantage un moyen de discuter entre soi. Ainsi, tel atelier observé, composé presque exclusivement d'Italiens et de Kenyans, les premiers ayant formé une partie des seconds et l'ensemble s'apparentant à une entreprise de fidélisation des partenaires. Le forum permet aussi aux organisations à la fois militantes et bailleuses de fonds de mettre en place et de piloter des initiatives avec leurs partenaires. C'est le cas du Comité catholique contre la faim et pour le développement (CCFD) qui a organisé des réunions avec ses « partenaires » africains, en marge du forum et pendant celui-ci, pour qu'ils rédigent un texte d'interpellation sur la politique africaine de la France, initiative des ONG françaises mais nécessitant un positionnement africain. Le forum est aussi une « vitrine » des activités des ONG bailleuses du Nord qui promeuvent les activités de leurs partenaires : des ONG africaines de défense de la cause des femmes ont ainsi présenté leurs activités au FSM avant une réunion programmée à Nairobi après le Forum pendant laquelle la ré-attribution de bourses par le principal bailleur était à l'ordre du jour. Cette relation objective de patronage n'est cependant plus assumée par tous les acteurs du Nord : beaucoup insistent sur la nouvelle donne que constitue l'altermondialisme au sein duquel les relations Nord-Sud auraient changé ${ }^{21}$. Malgré cette insistance, la dépendance évidente de la participation des délégués africains au bon-vouloir de leurs partenaires est vivement critiquée car elle reflèterait la perpétuation d'une domination impérialiste ${ }^{22}$. En même temps, cette intervention est réclamée par d'autres personnes qui estiment n'avoir aucun autre moyen de se rendre dans ce type d'événement

20. Dès 2002, lors de la préparation du premier Forum social africain de Bamako et du deuxième FSM de Porto Alegre, les organisations africaines bénéficient de financements de la coopération française, de l'UNESCO, de Novib, Oxfam et du CCFD. Voir Sulmont R., Forum social africain : émergence d'une mobilisation transnationale africaine, mémoire de DEA, Sciences Po Paris, 2004.

21. Massiah G., cité dans le compte rendu, op. cit.

22. "Africa: WSF - it was a meeting of NGOs, not the masses", East African Standard, 28 janvier 2007, rédigé par Tajudeen Abdul-Raheem, deputy director for Africa for the UN Millennium Campaign. Le problème s'observe aussi quand des délégués africains dépendent du financement du FSA. 
international. Entre les deux positions, se déploient des stratégies de ruse autour de cette dépendance matérielle : c'est le cas de ces militants tellement bien intégrés qu'ils offrent leurs financements multiples à des militants moins connectés, ou à l'inverse de ceux qui ne se lassent pas d'être les représentants de leur organisation et se forgent le statut d'unique interlocuteur pour la sphère militante altermondialiste. Cette autonomie des militants est réaffirmée dans les ateliers où les Africains - et les délégués du Sud en général - sont majoritaires. Au-delà du discours, elle passe aussi par la multiplicité des sources de financement, et notamment l'insertion des groupes dans divers réseaux transnationaux, gage d'une dépendance «maîtrisée » face à des bailleurs qui peuvent décider de ne plus soutenir ces initiatives ${ }^{23}$.

Pour les délégués interrogés qui ne viennent pas d'Afrique de l'Est, la participation au FSM fait partie de la « routine » militante ${ }^{24}$. Une majorité des militants d'Afrique de l'Ouest, du Centre ou du Sud, est déjà allée en Europe, aux Etats-Unis, en Inde ou en Amérique latine, et a voyagé en Afrique, pour des conférences, des séminaires, des formations ${ }^{25}$. Pour une minorité d'entre eux, le voyage est une composante essentielle de leur travail, une militante de la cause des femmes assurant: "je sors d'un avion pour aller dans un autre». La présence de ces militants déjà connectés peut s'expliquer par les coûts induits par les déplacements et le fait que ces derniers soient couverts par des partenaires du Nord, qui connaissent déjà le délégué invité ou, tout du moins, son organisation. Peu de place est laissée au renouvellement militant : les voyages entraînent les voyages à travers la construction de réseaux, mais aussi, plus prosaïquement, du fait des visas. Parmi ces militants voyageurs, certains avaient déjà une expérience internationale avant même leur entrée dans la sphère altermondialiste, notamment à travers des études à l'étranger. Mais pour la plupart, c'est bien l'insertion dans des réseaux transnationaux qui génère les voyages. L'internationalisation militante ne se résume pas à un effet de dispositions antérieures et, notamment, de l'appartenance à des élites sociales déjà internationalisées : elle apparaît tout autant comme une conséquence du militantisme. Dans le cas des délégués africains au FSM, ce sont les militants est-africains qui ont pu profiter de la proximité géographique du forum pour faire leur entrée dans la sphère altermondialiste et engranger des

23. Cela a été la stratégie du FSA qui, perdant le soutien financier du CCFD, première grande ONG à avoir soutenu la dynamique africaine, après le premier forum de Bamako a réussi à se perpétuer grâce à l'élargissement et la diversification de ses sources de financement. Voir Sulmont R., op. cit.

24. Sur les parcours d'internationalisation des militants africains au FSM, voir Siméant J., «S'engager dans l'internationalisation. Trajectoires de militants africains au FSM », communication présentée à la journée d'étude : «Un FSM en Afrique - Nairobi 2007. Causes africaines et extraversions militantes ", op. cit.

25. Sur les ressources sociales de l'accès à l'international, notamment dans l'espace altermondialiste, voir : Gobille B., «Les altermondialistes : des activistes transnationaux », Critique internationale, $\mathrm{n}^{\circ} 27$, avril-juin 2005, pp. 131-145; Cheynis E., « L’altermondialisme au prisme marocain », ibid., pp. 177-191. 
contacts. Certains sont venus chercher une protection : c'est le cas des militants ougandais et kenyans de la cause gaie et lesbienne dont quelques séminaires avaient pour objectif de rendre la cause visible, mais aussi de constituer des réseaux transnationaux permettant de protéger les militants par l'intervention d'organisations internationales.

\section{Trajectoires d'altermondialistes africains}

Malgré leur diversité (hommes, femmes, trentenaires, sexagénaires, venant de toutes les régions et de toutes les confessions d'Afrique), les trajectoires militantes de ces délégués ont cependant quelques caractéristiques communes, notamment en termes d'origine sociale, de parcours scolaire ou de rapport à la religion 26 : niveau d'étude et socialisation politique élevés qui facilitent la compréhension et la formulation des griefs, rapport critique à la religion, expression d'une appétence au débat...

$\mathrm{Si}$, pour beaucoup des militants africains interrogés, le milieu social d'origine n'est pas élevé, voire modeste, certaines caractéristiques distinguent leur famille de leur milieu environnant, notamment parce que les parents (en général le père) sont de petits fonctionnaires ayant pu encourager leurs enfants à aller à l'école. L'école, en effet, constitue souvent le creuset d'un apprentissage de la prise de parole au nom de tous : un grand nombre de militants interviewés sont d'anciens délégués de classe ou responsables d'associations de jeunes. Ces personnes engagées au nom des plus pauvres découvrent, par leur travail, les réalités du terrain et n'en sont pas les porte-paroles «naturels». Parce qu'ils étaient mieux lotis, ou parce qu'ils habitaient en ville, ils disent avoir pris conscience des difficultés sociales des femmes ou des paysans par leur travail. Une minorité de délégués africains interrogés provient de familles aisées et de catégories socioprofessionnelles supérieures. C'est le cas d'un certain nombre d' «élites mondialisées » des ONG africaines, telles qu'on les retrouve notamment au sein du FSA. En plus de ces origines sociales, le rapport tolérant des familles aux engagements souligne l'importance de l'approbation des proches dans la poursuite d'engagements contraignants en termes de vie familiale et sociale. Les militantes de la cause des femmes insistent fréquemment sur le soutien accordé par leurs maris (par exemple après des conflits), indispensable à la réussite de leur entreprise militante autour de pratiques culturellement délicates, comme l'excision.

Les délégués africains interrogés au FSM, ceux entre autres qui ne sont pas originaires du Kenya et d'Afrique orientale, sont majoritairement diplômés de l'enseignement supérieur. Juristes, enseignants du secondaire et du supérieur, ingénieurs, fonctionnaires, ils sont nombreux à avoir pu suivre des études

26. Pour une enquête sociographique sur l'altermondialisme européen, voir Agrikoliansky E., Sommier I., op. cit. 
supérieures, le plus souvent dans une université africaine. Etre proche des organismes de développement liés à l'international permet parfois de parfaire ses études à travers des bourses octroyées par les donateurs extérieurs, ou de multiplier les formations ponctuelles. Cette multiplication des formations s'insère dans une dynamique de professionnalisation du militantisme. Et, du fait du caractère de plus en plus professionnalisé du militantisme, au sein des ONG notamment, ce dernier tend à devenir un moyen de combiner engagement personnel et activité professionnelle, rémunératrice et conforme au niveau scolaire. Cette imbrication entre militantisme et profession est une tendance générale qui ne devrait pas être stigmatisée par une sociologie un peu brutale des rétributions du militantisme : pour certains militants interrogés, le travail au sein de structures militantes se fait d'abord sur le mode du bénévolat, avant d'arriver à se faire salarier.

Dernier élément, le rapport des militants africains interrogés à la religion. La dimension religieuse du forum de Nairobi a été soulignée comme une spécificité de ce FSM. Tous les délégués interrogés sur ce sujet affirment être croyants ${ }^{27}$, mais revendiquent une pratique réfléchie, voire distanciée, de leur religion, parfois même décalée : cette distance apparaît autant comme un effet du militantisme que comme un aspect de la socialisation primaire. Un militant de l'environnement kenyan affirme : "J'ai été élevé dans un milieu catholique. Je continue d'aller à l'église, mais je ne suis pas d'accord avec la plupart des choses qu'ils font là-bas; mais j'y vais quand même 28 ». Comme en écho, un militant congolais de la dette assure : «je suis un catholique rebelle 29 ». Encore plus explicitement, un militant de la cause LGTB en Ouganda adopte une pratique clairement en décalage avec la religion anglicane - et avec la religion en général - puisqu'il assiste aux offices d'un prêtre ougandais excommunié pour sa défense des homosexuels.

\section{Le militantisme africain internationalisé : un espace de tensions}

La division internationale du travail militant, à l'échelle continentale ou mondiale, multiplie par définition les possibilités de tensions que le discours militant essaie en partie de gommer, ou qu'il retraduit en oppositions idéologiques.

\section{Un altermondialisme africain ?}

Si l'Afrique est évoquée comme matrice de combat, les divisions et concurrences entre régions, organisations et personnalités ne manquent pas.

27. Une Sud-africaine néanmoins, militante contre la privation de l'eau, affirme avoir été éduquée dans la religion chrétienne mais être devenue a «non-religious socialist », entretien réalisé par L. Kariuki, 24 janvier 2007.

28 . Entretien par D. Connan et M.-E. Pommerolle, 22 janvier 2007, en anglais.

29. Entretien par J. Siméant, 21 janvier 2007. 
Mais, lors des présentations publiques, soit ces tensions continentales sont mises de côté par les délégués africains, soit les divisions sont imputées au Nord, qui les véhiculerait, telles un cliché.

[Question] : «Selon Christophe Aguiton, on peut déceler quatre tensions dans le FSA : entre anglophones et francophones, entre les Sud-Africains et les autres, entre la position plus "ONG" et la position plus "mouvements sociaux", entre le monde arabe et le reste de l'Afrique?

Ça c'est faux ! Je te dis que c'est faux, c'est les gens qui jugent à partir de leur propre perception mais c'est pas la réalité... Des tensions, elles existent mais c'est des tensions politiques... politiques... Ça n'a rien à voir avec anglophones... Ça n'a absolument rien à voir... elles ont leur source... Sur le plan politique... C'est des gens qui ont des divergences politiques... idéologiques même... C'est ça... Mais ça n'a rien à voir... Les Sud-Africains par exemple dont il parle... C'est des mouvements trotskistes... qui voulaient contrôler... le secrétariat... On a eu un débat très chaud à Addis-Abeba... Donc ça n'a rien voir avec... euh la langue... Heureusement nous avons surmonté ça... Nous sommes africains... Moi je me vois pas francophone pas plus que mon frère mon ami sud-africain n'est anglophone... Nous sommes africains... 30 "

Le FSA se tient pour la première fois à Bamako en janvier 2002. Sa naissance traduit alors une volonté d'autonomie des militants africains face à des initiatives cherchant à encourager la participation africaine au FSM, mais pilotées par des ONG du Nord (ATTAC ou le CCFD). Le FSA va néanmoins émerger grâce à des financements de la coopération française ${ }^{31}$. S'ouvrant progressivement au-delà de son premier cercle ouest-africain, le conseil du FSA, créé en 2004, est composé d'une quarantaine d'organisations venant de tout le continent. Cependant, des critiques de l'intérieur et de l'extérieur se font entendre. Elles émanent aussi bien de groupes initialement minoritaires dans cette dynamique, les anglophones, et plus particulièrement les Sud-Africains ${ }^{32}$, que de militants d'Afrique de l'Ouest ou centrale qui dénoncent l'approche choisie par le FSA. Ces derniers lui reprochent notamment d'entretenir des relations ambiguës avec les gouvernants africains et les institutions inter-gouvernementales comme l'Union africaine. Des critiques sur le fonctionnement du FSA, son opa-

30. Demba Moussa Dembele, Sénégal, économiste, membre du FSA, animateur du Forum africain des alternatives, coordinateur de la conférence internationale de Dakar pour l'annulation de la dette, 59 ans. Entretien par J. Siméant, 23 janvier 2007.

31. Sur la naissance du FSA, voir Sulmont R., op. cit.

32. Voir par exemple "Briefing document on the African social forum prepared for the South African delegates to the world social forum at the request of the South African world social forum preparatory meeting held on 7 January 2004", reproduit dans Sulmont R., ibid. 
cité, la concentration des décisions et son intrusion, parfois mal vécues, dans l'organisation du FSM de Nairobi se sont fait entendre. Outre ces concurrences pratiques, des divergences de conception à propos de la nature politique et stratégique de l'altermondialisme s'expriment au sein du FSA ${ }^{33}$. Deux clivages classiques se superposeraient au sein de l'altermondialisme : celui entre ONG et mouvements sociaux, et celui entre ceux qui conçoivent le FSM comme un «espace » et ceux qui y voient un «mouvement ${ }^{34}$. Ces tensions au sein du FSA révèlent surtout une lutte entre des réseaux et des traditions de mobilisation différentes, et notamment entre certains francophones, qui ont pris l'initiative, et les anglophones, et plus particulièrement les Sud-Africains initialement laissés de côté. D’autres facteurs se rajoutent au manque de relations entre les organisations et mouvements de ces régions qui ne partagent pas la même langue. Il semblerait qu'à l'origine, souhaitant écarter les ONG anglo-saxonnes du processus FSM, les initiateurs du FSM, brésiliens et européens, puis ceux du FSA, aient, par ricochet, mis de côté leurs partenaires d'Afrique australe qui, pour certains, entretiennent des relations privilégiées avec les ONG britanniques ou américaines comme $O x \mathrm{fam}^{35}$. En réalité, les tensions au sein du FSA et les critiques à son encontre dérivent surtout d'une concurrence vive entre des espaces militants régionaux perçus comme de potentiels concurrents dans le leadership de l'altermondialisme sur le continent.

L'insertion des Sud-Africains dans le processus du FSA suscite des réticences face à leur volonté de domination supposée sur l'espace militant africain, et du fait de leur savoir-faire militant et de leur radicalité, tous deux acquis dans la lutte anti-apartheid et renouvelés par les luttes anti-libérales déployées contre le gouvernement de Thabo Mbeki ${ }^{36}$. Cette tentation hégémonique, que certains comparent au positionnement politique de l'Etat sud-africain sur le continent, se manifeste surtout par la distinction des actions sud-africaines dans le cadre du FSM. Ils sont aux avant-postes des manifestations pour la gratuité des droits d'entrée, ils sont les seuls Africains à préparer l'assemblée des mouvements sociaux ${ }^{37}$, ils se font remarquer dans les ateliers à travers une radicalité exprimée (poing levé, «comrades» et Amandla) ${ }^{38}$. La volonté d'hégémonie sud-africaine évoquée par les autres délégués africains doit cependant être nuancée, dans la mesure où, au sein même de l'espace militant sud-africain, des tensions se font

33. Hlatshwayo M., «Le Forum social africain : entre radicaux et réformateurs », in Amin S., Houtart F., CETRI, Mondialisation des résistances. L'Etat des luttes 2004, Paris, Syllepse, 2004, pp. 221-226.

34. Ngwane T., "WSF 2007 in Africa must build a mass movement against capitalism", disponible sur : http://www.cadtm.org/article.php3 ?id_article=1168 (consulté le 22 novembre 2007).

35. Sulmont R., op. cit.

36. Cette crainte d'une hégémonie sud-africaine se lit encore plus explicitement dans les forums sociaux régionaux d'Afrique australe : voir Dwyer P., Larmer M., "'Four wheel drives and burning tyres': civil society and social movements in Southern Africa”, paper delivered at the International Sociology Association World Congress, Durban, 27 juillet 2006.

37. Observations par N. Haeringer.

38. C’est-à-dire la phraséologie de la lutte anti-apartheid. 
jour. En effet, le clivage est désormais important entre d'un côté les mouvements communautaires « de masse » se mobilisant contre les privatisations de l'eau et de l'électricité, pour certains d'obédience trotskiste, et s'opposant à l'African National Congress (ANC) et, d'un autre côté, les grandes ONG menant des campagnes internationales comme la Treatment Action Campaign, dont le succès a confirmé la réputation sud-africaine en matière de mobilisation ${ }^{39}$.

\section{ONG et mouvements sociaux}

Le clivage entre ONG et mouvement sociaux, fondé sur la nature des acteurs de l'altermondialisme, agite le processus du FSM depuis ses débuts. Sur le plan sociologique, on pourrait discuter cette opposition routinière et peu claire (des ONG participent aux mouvements sociaux...), mais elle fait immédiatement sens pour les acteurs. S'opposeraient ainsi d'un côté les ONG, structurées, dépendantes de fonds extérieurs, dirigées par des élites intellectuelles réformistes, et de l'autre des mouvements sociaux, syndicats ou autres, reflétant les aspirations populaires et visant à une véritable transformation sociale et politique. Depuis le début, cette tension s'est manifestée au FSM par une tentative de démarcation nette entre les deux tendances, à travers la mise en place d'une assemblée des mouvements sociaux qui se déroule traditionnellement à la fin du FSM : l'objectif est alors de délimiter un espace distinct, mais aussi de montrer que le FSM peut aboutir à une déclaration commune, prélude à une mobilisation. Lors du FSM de Nairobi, le clivage a été exposé de manière très nette lors des manifestations conduites par des leaders du mouvement contre le comité local d'organisation à propos des conditions d'entrée au forum ${ }^{40}$. Cette exacerbation peut se comprendre par l'acuité de ce clivage originel au sein de l'altermondialisme africain et par l'histoire du monde associatif depuis les années 1980 sur le continent, marquée par l'émergence d'une « société civile » soutenue par les ONG internationales, mais aussi par un contexte de revendications démocratiques et un besoin de pallier les carences sociales de l'Etat ${ }^{41}$.

Alors que les syndicats sont, à cette époque, laminés par les partis uniques et par des transformations importantes du monde du travail, ce sont effectivement les ONG, soutenues par l'étranger, qui capitalisent les revendications exprimées

39. Zuern E., «La pauvreté en débat. Marginalité et démocratie constitutionnelle en Afrique du Sud », Politique africaine, n`103, décembre 2006, pp. 27-45. Ces divisions se sont exprimées fortement lorsque l'Afrique du Sud avait été envisagée pour accueillir le FSM : trop divisées, les organisations sud-africaines avaient préféré renoncer à en être les hôtes. Par ailleurs, cet apparentement de la TAC à la catégorie «ONG » n'est pas cependant l'auto-catégorisation choisie par ses membres qui préfèrent se qualifier de "social movement" (entretien de M. Ndour avec la chargée des campagnes à la TAC, 23 janvier 2007). Ces processus de désignation font partie du rapport de force.

40. Des prises de parole sur ce sujet lors du Conseil international du FSM des 25 et 26 janvier, à Nairobi, sont nombreuses.

41. Voir Igoe J., Kelsall T. (eds.), Between a Rock and a Hard Place: African NGOs, Donors and the State, Durham, Carolina Academic Press, 2005. 
au début des années 1990 et s'imposent comme les interlocuteurs des Etats et des groupes extérieurs. Néanmoins, critiquées de toutes parts à partir de la fin des années 1990, elles tentent de retrouver une légitimité en faisant appel aux organisations de base. Une jonction se fait alors entre des groupes de paysans ou d'habitants de bidonvilles, des leaders communautaires cooptés et des ONG, permettant à celles-ci de développer un discours de légitimation, mettant l'accent sur les mouvements sociaux et sur leur base communautaire. Si les ONG acceptent la critique, notamment celle de leur dépendance face à l'extérieur, et appellent à la diversité du mouvement altermondialiste, elles rappellent néanmoins qu'elles ont comblé un vide après l'effondrement des syndicats. Ces derniers ont cependant marqué leur retour lors du FSM de Nairobi. Largement soutenus pas les réseaux internationaux, les syndicalistes africains présents reconnaissent leurs difficultés à mobiliser et admirent les mobilisations des ONG et mouvements sociaux qu'ils jugent «modernes ». Le renouveau de l'internationalisation des syndicats africains ainsi que la tendance à l'importation des méthodes des ONG semblent marquer leur inscription dans le processus altermondialiste africain et brouiller les clivages «ONG »/ « mouvements sociaux ». Au sein du FSM, la tendance « mouvements sociaux » demeure d'ailleurs largement organisée et défendue par des délégués non africains, comme l'a montré l'Assemblée des mouvements sociaux tenue le dernier jour du forum et rassemblant près de 500 personnes, selon nos estimations ${ }^{42}$. Alors que la consigne au départ est donnée de privilégier les interventions de représentants africains, et notamment de femmes africaines qui seront effectivement majoritaires, l’audience compte une minorité de délégués africains.

\section{Parler au nom de l'Afrique : doubles contraintes et censures}

Ce forum mondial aura été, plus que d'autres, l'occasion d'observer la façon dont les militants africains, mais aussi le reste du monde, parlent de l'Afrique dans un événement militant internationalisé. Cela semblait presque évident : quelle meilleure preuve des méfaits de la mondialisation et du capitalisme que la situation actuelle de l'Afrique ? Mais précisément parce que l'Afrique apparaît comme un continent victime, se mobiliser en son nom n'allait pas sans ces doubles contraintes propres à tous les mouvements qui entendent à la fois dénoncer la domination et se montrer capables d'action (agency) ${ }^{43}$, et à tous les acteurs qui s'internationalisent mais revendiquent le droit à représenter authentiquement leur base.

\section{Capacité d'action et domination}

Comment critiquer des situations de misère sans tomber dans le misérabilisme ou faire l'aveu de son impuissance ; comment célébrer, à l'inverse, sa

42. Les principaux organisateurs de cette assemblée sont: Marche mondiale des femmes, CADTM, Focus on the global South et des personnalités comme Christophe Aguiton (de la LCR).

43. Gamson W., Talking Politics, Cambirge, Cambirdge University Press, 1992. 
capacité de lutte et d'action sans donner dans le populisme, en ignorant ses difficultés réelles et en faisant fuir de possibles soutiens par une revendication sourcilleuse d'autonomie culturelle et politique ? On reconnait ici les dilemmes classiques du misérabilisme et du populisme croisés ${ }^{44}$. A Nairobi, l'Afrique était à la fois agie et actrice - en actes en en paroles.

Ces contraintes pèsent aussi bien sur les militants africains que non africains. Ainsi, le bilan du FSM variait-il en fonction de ces autocensures : les membres du FSA ou les représentants du comité d'organisation kenyan considéraient que la critique de l'organisation portait sur des éléments véniels, qu'elle négligeait les difficultés propres à l'Afrique, voire révélait le " néocolonialisme » de certains militants du Nord. Beaucoup de représentants d'ONG internationales, plus familières des difficultés du travail en Afrique, trouvaient, parfois non sans paternalisme, que " pour l'Afrique », c'était une réussite... A l'inverse, les critiques les plus virulents de l'organisation, qu'ils viennent du Nord ou du Sud, étaient ceux pour lesquels la dimension africaine du forum n'était pas un enjeu (ou, à l'inverse, ceux qui entendaient dénoncer les prétentions de «l'élite» du FSA, comme les militant liés au CADTM). Pour eux, dès lors qu'un pays du Sud avait réussi à organiser un FSM avec une bonne participation populaire (comme à Mumbaï), il n'y avait pas lieu d'épargner les organisateurs kenyans.

Le risque de se faire reprocher leur paternalisme est intériorisé et anticipé par beaucoup de militants du Nord. Au forum, ces derniers se risquaient rarement à critiquer les gouvernements africains. Tout au plus en faisaient-ils les marionnettes ou les complices des gouvernements du Nord, laissant aux Africains le soin de critiquer, ou non, leurs gouvernements. C'est ainsi que dans l'atelier Extractive and local livelihoods, les militants nigérians du Delta du Niger ont accusé le gouvernement fédéral d'être complice des compagnies pétrolières, tandis que les participants occidentaux dénonçaient les grandes puissances américaines et britanniques. Si l'anti-impérialisme est une des idéologies les mieux partagées au FSM, même la sollicitude dont les militants du Nord sont susceptibles de faire preuve à l'égard des Africains victimes de l'impérialisme peut être vécue sur le mode du paternalisme, d'où cette forme de division internationale de la critique militante.

Il n'y a bien sûr pas une façon unique d'envisager l'Afrique de la part des militants du Nord présents à Nairobi, ne serait-ce que parce que se côtoyaient des strates militantes très diverses : membres d'organisations d'aide au développement, trotskistes opposés à la guerre, militants chrétiens contre la dette, féministes mainstream ou radicales, anticolonialistes de la première heure...

44. Grignon J.-C., Passeron J.-C., Le Savant et le Populaire. Misérabilisme et populisme en sociologie et en littérature, Paris, Gallimard/Seuil, 1989. 
De surcroît, le comportement des militants du Nord, leur ethnocentrisme pratique ou théorique n'est pas toujours en lien direct avec leurs préférences idéologiques, mais a beaucoup à voir aussi avec leur familiarité à l'égard du continent, voire avec leur socialisation et leur position sociale. En revanche, un des aspects frappants des forums en général et de ce forum en particulier est la réactivation d'une idéologie tiers-mondiste dont les années 1980 avaient marqué le fort déclin et la remise en question. Cette forte présence tient sans doute à l'espace de convergences entre militants africains et non africains que constituent ces diverses déclinaisons du tiers-mondisme : selon les ateliers, on entendait parler de la critique de la dépendance (parmi les «stars» du forum, on pouvait remarquer Samir Amin,), du tiers-mondisme (à travers le Centre d'études anti-impérialistes, le CEDETIM), de la théologie de la libération (le chanoine Houtard, un de ses grands théoriciens, était présent au forum), de l'anticolonialisme (l'espace Franz Fanon fut particulièrement actif), ou enfin de l'afrocentrisme, fût-il d'origine afro-américaine (Malcom X Grassroots Movement) ou africaine (voir les nombreuses références à Cheikh Anta Diop).

Tout au long du FSM, une forme - certes modérée - d'afrocentrisme, est donc apparue comme un des procédés permettant de faire la preuve d'une capacité d'action et de mobiliser une fierté identitaire, tout en dénonçant le sort fait au continent, façon de lier ce que Gamson identifie comme les trois composantes centrales de l'action collective : injustice, agency, identity.

Un premier aspect de cet afrocentrisme consistait classiquement à rappeler ce dont l'Afrique peut être fière : célébration de grands intellectuels africains, retour sur les grandes figures historiques des indépendances et luttes africaines, inscrites jusque dans le nom des espaces du forum. La référence aux héros disparus de l'Afrique est révélatrice du rapport ambivalent des altermondialistes d'Afrique aux dirigeants africains : revendication sourcilleuse de la souveraineté des Etats africains d'un côté, dont ils n'ignorent pas qu'elle pourra être reprise par ces dirigeants eux-mêmes (ce qui fut historiquement le cas, de toutes les façons, quand l'anti-impérialisme était arrimé à un projet de construction nationale), mais critique des «marionnettes du Nord», de l'autre. La critique de la corruption des dirigeants africains se fait plus dans des situations non officielles, entre amis militants, du Nord ou du Sud (et pas seulement, même si c'est aussi un élément, par crainte de représailles une fois rentré au pays) que face aux militants du Nord dans des situations publiques. Elle est aussi le fait de militants «novices » (ici, les Kenyans, paysans ou squatters) qui n’hésitent pas à dénoncer clairement les responsables politiques, plutôt que celui des militants chevronnés qui ont adopté, depuis leurs débuts, une attitude ambivalente à l'égard de leurs dirigeants. Cette contrainte est certes propre à la plupart des critiques de la domination : que dire qui ne risque d'être utilisé par les adversaires ? Mais elle se renforce dans le cadre des revendications de type nationaliste ou anti-impérialiste, car critiquer les dirigeants africains peut fournir de nouveaux arguments aux institutions financières 
internationales, promptes à dénoncer la corruption et à encourager la « bonne gouvernance » à défaut de la démocratie. La dénonciation de la dette est très révélatrice de cette forme contrainte de la critique des dirigeants. L'exemple de la dette de la République démocratique du Congo, très souvent utilisé, s'explique sans doute par le fait qu'il permet de critiquer un ancien dirigeant africain, Mobutu, en expliquant que les Africains n'ont pas à payer la dette de l'illégitime dictateur mais, qu'à l'inverse, les pays du Nord sont redevables des horreurs commises par leurs ancêtres.

On retrouvait fréquemment à Nairobi cette forme de critique « latérale », qui consistait à dénoncer des coupables lointains ou encore des processus (les accords de partenariat économique, les institutions financières internationales, le Nord, l'impérialisme, l'héritage de la colonisation, etc.). Cela permet en effet d'endosser un registre critique, même lorsque l'on est le citoyen d'un régime peu démocratique, en conciliant ainsi un activisme aux contours modérés dans son espace national avec une radicalité de langage, fut-elle dirigée vers des ennemis extérieurs, malgré tout importante dans les cercles internationaux - et en laissant dans le flou la question de la responsabilité des dirigeants nationaux.

Un autre aspect de cette conciliation de l'agency et de la critique de la domination s'observe dans des prises de parole jouant à la fois sur l'affirmation de maux de l'Afrique et sur le refus que l'Afrique se résume à ces derniers. Le discours de Wahu Kaara, activiste kenyane, lors de la cérémonie d'ouverture du FSM, est révélateur de cette forme d'expression, face aux contraintes que pose un rappel des maux de l'Afrique qui voudrait ne pas sombrer dans le misérabilisme : elle consiste en un récapitulatif de ces maux, mais sur le mode de la dénégation et de la prétérition qui, jointe à un discours énergique, mobilisait effectivement une forme de capacité d'action :

" "Welcome to Wabu Kaara, the African revolutionnary!" ", lance le présentateur sur l'estrade. Wahu Kaara lance une série de "Karibu » et de "Welcome », et explique que Nairobi est accueillante. Sa harangue, au bord du hurlement, est suivie d'acclamations :

"Africa is not a dying continent!"
Africa is not a (bargain) continent!"
"Africa is not a poor continent!"
"Africa is not a dying continent!"
"Africa is not a continent of diseases!"
"Africa is not a continent of malnutrition!"
"Africa is a continent of human spirit!"

[...] It can be very sentimental and very emotional... very sentimental and very emotional because we are here in Nairobi to say that Africa is here and now to stay!" (Yeab!) 
La formation de cette agency africaine se traduisait aussi par la délimitation $\mathrm{du}$ « eux » et du « nous », façon de tracer aussi bien les frontières entre amis et ennemis qu'entre militants légitimes ou illégitimes à s'approprier la cause de l'Afrique. On retrouvait ce clivage (vous/nous/eux) dans nombre d'ateliers : le «nous » désignant l'Afrique et le « vous », le Nord. Cela pourrait paraître paradoxal dans un forum défini, selon sa charte, comme un espace «ouvert », comme une instance de coordination des mouvements de la société civile de tous les pays du monde. Rappeler ce clivage, c'est aussi tenter de prévenir le risque que les militants du Nord ne dominent la lutte en faveur du Sud ${ }^{46}$, d'où cette dimension très situationnelle $\mathrm{du}$ " nous/vous » : c'est souvent à l'occasion d'un débat où un militant blanc s'exprime trop longtemps, ou quand un militant africain fait remarquer avec amertume que les lieux où il doit loger ne sont pas aussi confortables que ceux des militants du Nord, ou encore qu'il n'a pas les moyens d'acheter la nourriture ou des boissons vendues à prix fort autour du FSM, que sont rappelées ces différences... ce qui peut très vite faire battre en retraite les Occidentaux.

Au FSM, l'identité africaine est donc sujette à transformations selon les interactions et les situations. Un militant du Nord qui idéaliserait la tradition africaine peut se voir reprocher, à juste titre, d'avoir une vision caricaturale et anti-moderne de l'Afrique, alors qu'à l'inverse, face à un public mixte, la référence aux traditions qui «marchent » est un classique, très influencé d'ailleurs par certains courants du développement. Il en est de même de la référence à la religion, qui peut, selon les situations, être alternativement dénoncée ou à l'inverse exposée comme exemplaire de "l'âme africaine ». Ces aspects posent donc la question des formes de légitimité revendiquées par les militants.

\section{Légitimisme culturel et autocensures}

La localisation de ce FSM créait une « injonction à l'Afrique » en son sein. Un espace de compétition pour parler au nom de l'Afrique s'ouvrait alors, entraînant diverses revendications de légitimité culturelle et d'authenticité, qui avaient pour enjeu le droit à la parole, parfois contre le Nord, parfois face aux autres militants africains.

Premier aspect de ce légitimisme culturel : une façon, classique, de revendiquer et de mettre en scène les traditions qui consiste à revendiquer, là

45. Observations par J. Siméant, 20 janvier 2007, cérémonie d'ouverture, Uhuru Park. Afin de conserver le rythme et la syntaxe excats de ce discours, nous avons choisi de ne pas le traduire.

46. Le clivage est alors plus Nord/Sud qu'Africain/Nord : lors d'un atelier Jubilee, un jeune Norvégien militant anti-dette se fit par exemple contredire par une militante équatorienne qui remettait en cause la parole de «cette personne du Nord, un jeune homme », 24 janvier 2007. 
encore, une agency et une identité issue de ses ressources culturelles propres ${ }^{47}$. Il peut s'agir de traditions et mémoires militantes (indépendances, la lutte contre l'apartheid). Cela renvoie aussi à des formes d'expression (chant, danse, etc.), souvent mobilisées entre les sessions, voire lors des inaugurations de ces dernières. Mais il s'agit plus largement d'une «culture africaine », idéalisée, peu explicitée, faite de recherche du consensus, de sens de la communauté, de rôle de la famille, des aînés et des liens sociaux traditionnels, ou encore de complémentarité des rapports homme/femme.

Cette référence à une tradition (ré)inventée n'a pas pour unique rôle de mettre à distance des militants du Nord perçus comme prompts à s'approprier des luttes. Elle joue également un rôle central dans les situations où le public est majoritairement africain et où l'on voit alors se déployer des stratégies d'attestation de représentativité. Ce qui semble en jeu dans cet espace internationalisé qui ressemble parfois tant à une énième conférence internationale, c'est surtout de ne pas pouvoir se faire reprocher de n'être pas africain (parce que «occidentalisé », coupé des Africains, voyageant trop...). C'est le cas de ces artistes kenyans, qui se produisent au Forum «au nom d'un sacrifice pour l'Afrique » et qui avouent ne pas aimer dire qu'ils «vivent » aux Etats-Unis où ils passent pourtant la majeure partie de leur temps, pour des raisons professionnelles. Si des accusations de ne représenter personne s'entendent parfois "off », contre certaines «stars » de l'altermondialisme africain, c'est que la transnationalisation du militantisme a pu contribuer à aggraver le fossé entre les militants les plus internationalisés, voire les plus dotés de ressources sociales et financières, et les autres. D'où cette insistance à montrer que l'on est bien africain, c'est-à-dire « culturellement » africain, qu'on ne reproduit pas des schémas colonialistes, que l'on n’a pas voyagé, que l'on est plus « rooted» que « cosmopolitan ${ }^{48}$.

Cette insistance à nier l'extraversion et l'internationalisation, on l'observe assez significativement dans les ateliers relatifs à la sexualité, aussi bien chez les militants africains qu'occidentaux. Chez ces derniers, cela se traduit aussi par une volonté de ne pas se mettre en avant, de ne pas sembler imposer des codes qui seraient rejetés s'ils apparaissaient comme promus par le Nord.

L'atelier «Reclaiming our sexualities» du 22 janvier 2007, au Queer Spot, bénéficie du soutien organisationnel de l'ILGA (International Lesbian and Gay Association), matérialisé par la présence discrète de Stephen Barris, qui se cantonne à la traduction de l'espagnol à l'anglais. L'enjeu est manifestement, pendant tout le

47 . Hobsbawm E., Ranger T., The Invention of Tradition, Cambridge, Cambridge University Press, 1983.

48. Tarrow S., "Rooted cosmopolitans: transnational activists in a world of states", Communication au séminaire "Contentious Politics: Identity, Mobilization and Transnational Politics”, Amsterdam School of Social Research, 6 mai 2003. 
Forum, de minorer la présence des Blancs sur cette thématique, comme le reconnait lui-même Barris dans son compte rendu rédigé après le FSM ${ }^{49}$. C'est dans ce seul atelier que nous avons observé la mobilisation de la thématique post-coloniale - et que nous avons le plus entendu parler de ce qui est «vraiment» africain ou de «Mother Africa»: la référence au reproche de l'homosexualité comme "non africaine » apparaît comme un obstacle permanent à contrer. Le public est varié, avec une courte majorité africaine. Quatre des cinq intervenantes sont africaines, toutes anglophones : deux Sud-Africaines, une Ougandaise réfugiée en Afrique du Sud, une Nigériane. Une activiste et poétesse sud-africaine évoque l'utilisation de l'homophobie par les leaders post-coloniaux qui la dénoncent comme non africaine. Elle explique : "nous sommes là précisément pour reconquérir nos sexualités ». La Nigériane évoque les travaux qu'elle a menés sur l'homosexualité au Nigeria:

«Il y a des gens qui sont nés au Nigéria, qui vivent au Nigéria, qui n'ont jamais quitté le pays, et qui ont des relations avec des personnes de même sexe [début d'applaudissements]. En anglais, on dirait que ce sont des homosexuels. Mais il est difficile de trouver un mot en langues locales décrivant ce comportement car la colonisation, la christianisation et le jihad les ont censurées pour imposer le code moral qu'ils charriaient » (traduit de l'anglais)

Elle rappelle qu'il existe des termes traditionnels pour désigner ce type de relations, qu'on peut découvrir en interrogeant les gens âgés. La leader de la Coalition of African Lesbians brandit un ouvrage d'anthropologie qui collecte des histoires de vie et témoignages de lesbiennes en Afrique anglophone. Contre l'idée de non-africanité de l'homosexualité, elle appelle à se réapproprier une terminologie, à se réapproprier le langage de la tradition qui permette de « dire à nos communautés ce qu'on est sans passer par le langage colonial».

La même manière de parler de ce qui est vraiment (ou pas du tout) africain se retrouve dans des ateliers correspondant davantage au monde de l'aide internationale et des financements des ONG du Nord, mais qui touchent aussi à la sexualité, comme dans le cas de cet atelier intitulé «Developing alternatives in the Panafrican fight against HIV and AIDS », animé par le salarié ougandais d'une grande ONG du Nord.

49. "Je reste derrière la scène : un Européen sur scène, blanc de surcrô̂t pour parler au nom des gays et des lesbiennes africains discréditerait l'intervention. »:

http://www.tetu.com/rubrique/mag/mag_dossier_detail.php?id_dossier=246, consulté le 22 novembre 2007. 
Le chairman, chargé des programmes HIV pour Action Aid, joue à merveille de la référence à la culture africaine et du refus de se laisser imposer quoi que ce soit par le Nord, tout en défendant l'idée que les traitements fonctionnent, que la prévention importe, que l'abstinence est un vou pieux, etc. Dans sa harangue, il interpelle le 10 Downing Street, la Maison-Blanche, Bruxelles... Il interpelle des citoyens du Nord :

«Il faut qu'on vous écoute, et qu'ils [les dirigeants] soient accountable! [...] et ceux qui rentrent [sous-entendu au Nord] vérifiez comment votre argent est utilisé plutôt que de financer des thèses! Soutenez des outils et des stratégies qui marchent : et même si vous achetez des condoms ça ne veut pas dire que je les utiliserai !... "It's my culture!" $50 »$.

Le public, majoritairement africain, augmente en nombre tant l'orateur réjouit la salle, et manifeste son accord.

S’interroger sur la participation africaine à ce FSM permet donc de tenir une double ligne, consistant à la fois à examiner les conditions concrètes $\mathrm{du}$ militantisme transnational, et à envisager les mouvements sociaux comme des espaces de lutte sur le droit à la parole légitime (l'internationalisation compliquant encore cette réalité que connaissent aussi les mouvements s’inscrivant dans un cadre uniquement national) et, ici, la parole militante légitime sur l'Afrique. Ces deux aspects, bien qu'analytiquement distincts, ne sont pas vraiment séparables. Rentrer dans le détail des conditions concrètes de la protestation transnationale (une « sociologie du billet d'avion » en quelque sorte), c'est assez rapidement comprendre où passent les lignes de force, d'alliance mais aussi de domination dans l'espace de la protestation transnationale. Cela permet de comprendre comment, sans se limiter à en être le reflet, certains affrontements idéologiques sont une façon de retraduire, dans le langage militant, des réalités qui correspondent aussi à des antagonismes de position sociale, à l'échelle nationale ou internationale. Cette étude matérielle et symbolique du FSM montre également à quel point cet événement protestataire est loin de l'image d'une société civile mondiale égalitaire. Elle souligne néanmoins qu'il est possible, pour les nouveaux arrivants, les outsiders et les dominés de remettre en cause, ne serait-ce que verbalement, ces relations inégales. La seconde étape de cette recherche consistera à examiner comment les nouvelles hiérarchies et formes de légitimation créées dans cet espace transnational sont utilisées et re-traduites dans les contextes nationaux. 